\title{
STATISTICS FOR PATCH OBSERVATIONS
}

\author{
K. L. Hingee $\mathrm{a}^{\mathrm{a}, \mathrm{b} *}$ \\ ${ }^{\mathrm{a} C S I R O}$, Underwood Ave, Floreat, Perth, Australia \\ ${ }^{\text {b }}$ School of Mathematics \& Statistics, University of Western Australia, Stirling Highway, Perth, Australia - \\ kassel.hingee@research.uwa.edu.au
}

Youth Forum

KEY WORDS: random closed sets, landscape pattern indices, land cover, thematic maps, spatial statistics

\begin{abstract}
:
In the application of remote sensing it is common to investigate processes that generate patches of material. This is especially true when using categorical land cover or land use maps. Here we view some existing tools, landscape pattern indices (LPI), as non-parametric estimators of random closed sets (RACS). This RACS framework enables LPIs to be studied rigorously. A RACS is any random process that generates a closed set, which encompasses any processes that result in binary (two-class) land cover maps. RACS theory, and methods in the underlying field of stochastic geometry, are particularly well suited to high-resolution remote sensing where objects extend across tens of pixels, and the shapes and orientations of patches are symptomatic of underlying processes. For some LPI this field already contains variance information and border correction techniques. After introducing RACS theory we discuss the core area LPI in detail. It is closely related to the spherical contact distribution leading to conditional variants, a new version of contagion, variance information and multiple border-corrected estimators. We demonstrate some of these findings on high resolution tree canopy data.
\end{abstract}

\section{INTRODUCTION}

Statistical analysis of images can be grouped into two main branches (Molchanov, 1997): (a) describing/classifying an observed scene or (b) considering the scene to be generated by a random process and inferring properties of this process. We are concerned mostly with the latter, and especially those processes observed in remotely sensed maps of categorical variables. Such analysis occurs when comparing different regions, comparing the same region at different times, gaining understanding of random processes (e.g. spatial dependence), or model fitting.

A random closed set (RACS) is a generic framework for modelling randomness in processes that generate spatial patterns of patches. It encompasses common models in remote sensing, such as those derived from Markov random fields and Gaussian random fields, and a wide range of other models (e.g. germ-grain models, birth-growth models, fibre processes, and tessellations). Markov random fields excel at contextual investigations, but have difficulty describing geometrical properties (Descombes, 2012). Gaussian random fields, completely determined by their mean and covariance (Chiu et al., 2013), can only capture first and second order characteristics of a process. Other RACS models can describe complex geometrical shapes and infinite-order characteristics (Descombes, 2012). These models reveal new methods for describing the geometry of random scenes such as contact distributions (Baddeley and Gill, 1994) and tangent point analysis (Barbour and Schmidt, 2001).

These tools have been combined with remote sensing to model uncertain object boundaries (Zhao et al., 2009), meteorological features (Cressie et al., 2012), fine-scale grass

\footnotetext{
${ }^{*}$ Corresponding author
}

patterns (Sadler, 2006) and urban tree locations (Rossi et al., 2015). The RACS framework has also been used to test the spatial dependence of beetle infestation (Kautz et al., 2011) and the relationship between tree deaths and bore locations (Chang et al., 2013). Numerous other examples exist (Descombes, 2012).

We examine parallels between non-parametric RACS summary functions and landscape pattern indices (LPI). To the author's knowledge this is the first published discussion on this topic. A RACS framework leads to more rigorous treatment of landscape pattern data and improved understanding of LPI behaviour, including border corrections, resolution robustness and conditional probabilities. In the next section we introduce RACS theory in more detail, with a focus on concepts relevant to remote sensing applications. Subsequently Section 3 discusses RACS and LPI. Finally Section 4 describes an application of nonparametric RACS estimators to maps of tree canopy.

\section{RANDOM CLOSED SETS AND REMOTE SENSING}

For brevity we omit many technical details and the full generality of RACS. These details can be found in a number of texts (Molchanov, 2005; Chiu et al., 2013). A RACS is any random process that generates a closed set. A closed set in Euclidean space is any set for which all points on the edge of the set are also in the set. A RACS, $X$, is then any process that maps from some state space $\Omega$ to closed subsets of Euclidean space

$$
P(X(\omega) \text { is closed })=1 .
$$

This is similar to the familiar definition of a random variable, except each realisation is a closed subset of space. Figure 1 contains two example realisations of RACS. A RACS 
produces categorical maps if its realisations are closed 2D regions. This definition allows only two classes, inside $X$ or not inside $X$, but can be generalised to multiclass realisations (Molchanov, 1984; Ayala and Simó, 1995; Kautz et al., 2011).
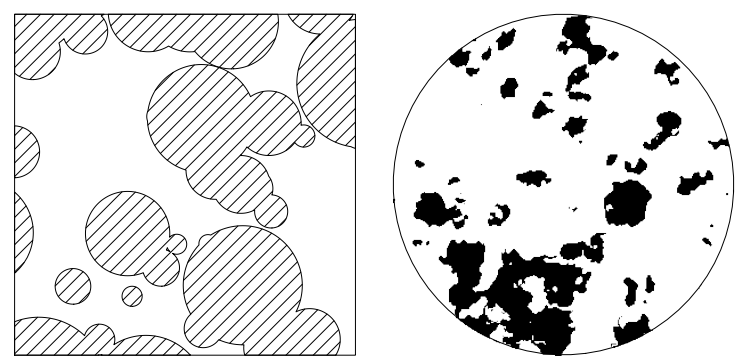

Figure 1: Example realisations of RACS. Left: A Boolean model (Stoyan and Mecke, 2005) observed in a rectangular region; hatched regions denote locations inside the random set. Right: A map of tree canopy which can be represented as a realisation of a RACS. This is also an example of the observations used in Section 4.

As an example the pattern of mould on the surface of an old slice of bread can be modelled as a RACS. The mould pattern may have some systematic properties (e.g. patch size) but the precise location, size and shape of the mould is unpredictable. For this RACS a single realisation is the pattern of mould on a single slice of bread.

The mathematical field underlying the statistical investigation of random scenes is known as Stochastic Geometry (Molchanov, 1997). It is the study of statistical methods for geometrical patterns (Matheron, 1975; Chiu et al., 2013). Historically an important application has been the inference of $2 \mathrm{D}$ or $3 \mathrm{D}$ properties (e.g. properties of rocks) from lower dimensional samples (Baddeley and Jensen, 2004). This application area, known as stereology, has developed tools for inference from 0D samples (e.g. pixels) and from images (2D samples).

Often it is the case in remote sensing of the Earth that only one realisation of the process is available. For example the generation of a native forest, including all its past disturbances, is usually seen only once at each location. We can mitigate this issue by assuming that the statistical properties of the process are similar at different locations and that the dependence between distant locations is small. Thus observations of different regions act like multiple realisations. These sort of assumptions are common in spatial analysis.

The strongest similarity assumption is stationarity which assumes the statistical properties of a process are translation invariant. In other words the probability of any closed, bounded set $K$ intersecting $X$, written $P(K \cap X \neq \emptyset)$, is independent of translations of $K$; the probability depends only on the shape and size of $K$. As an example consider the centre of the mouldy bread slice at high magnification so that we may ignore the border of the bread and assume that the process is stationary. This stationarity assumption implies that, before looking at the bread, the probability that any particular location is mouldy $P(x \in X)$, known as the coverage probability, is the same for all $x$. It also means that the probability that a circular region contains mould depends only on the radius, which leads to the spherical contact distribution (SCD) discussed later.
These examples correspond to intersecting with a set $\{x\}$ and a disc respectively. The stationary assumption has been common in landscape ecology (Fortin et al., 2003).

There are a number of summary functions that are well defined for stationary RACS. A summary function describes particular properties of a RACS, typically as a probability or the expectation of some quantity. Examples are the coverage probability, the spherical contact distribution and the expected perimeter-length per unit area. They have been used to fit parameters and uniquely determine parameters for some model classes (Hug et al., 2002).

Once we have observed something about the mould, near location $a$ say, then the conditional probability of an event near $b, P$ (event near $b \mid$ event near $a)$, is not usually translation invariant because it depends on the location of $b$ relative to $a$. In general RACS observations at different locations can be very dependent.

A stationary process is mixing if, as $b$ gets further away from $a$, the probability, $P$ (event near $b \mid$ event near $a$ ), approaches the value that it would have assuming independence. Stationary and mixing processes are ergodic (Daley and Vere-Jones, 2008, $\$ 12.3$ ) which means spatial averages of large windows may be used instead of averages across multiple realisations. For example if the bread mould process is ergodic then the probability of a particular location being mouldy can be estimated by the proportion of mouldy locations in a large window; multiple slices of bread are not needed. Note that in practice the stationarity assumption requires observations in windows small enough that changes in the environment (e.g. a distant change in soil type) can be ignored whilst estimates using the ergodic property require large windows. The window size is then a compromise between estimator accuracy and the credibility of the stationarity assumption.

\section{AN APPLICATION TO LANDSCAPE PATTERN INDICES}

Landscape ecologists study both the effect of spatial pattern on ecological processes wherein spatial pattern is considered a covariate and the effect of ecological processes on spatial pattern wherein the spatial pattern is considered a response or symptom of the ecological process (Fortin and Agrawal, 2005). Frequently this requires comparing patterns observed in remotely sensed maps of land cover type.

LPIs are numerical descriptions of spatial configuration that are commonly used in landscape ecology (Lustig et al., 2015). Current LPIs have proved sensitive to resolution and boundary effects (Kupfer, 2012). Many LPIs are also difficult to interpret (Schröder and Seppelt, 2006) and highly correlated with other LPIs (Cushman et al., 2008; Schindler et al., 2008; Turner, 2005). There have been numerous calls for more rigorous statistical interpretation of LPIs (Lustig et al., 2015; Dramstad, 2009; Wang and Cumming, 2011) and some calls for more process-based metrics (Fortin et al., 2003; Remmel and Csillag, 2003). RACS provide a generic probabilistic framework that allows statistical interpretation, process-based descriptions and rigorous study of LPI behaviour.

The most popular software package for the calculation of LPIs is FRAGSTATS (McGarigal, 2015) and many other packages incorporate LPIs that are conceptually derived 
from FRAGSTATS (VanDerWal et al., 2015). Within the collection of metrics available in FRAGSTATS we have noticed that:

1. The percentage of landscape index is an estimator of coverage probability

2. The edge density index is a (potentially biased) estimator of an identically named concept in RACS theory.

3. The percentage of core area is an estimator of the core probability which is related to the spherical contact distribution for a RACS.

4. The description of the radius of gyration given by (Keitt et al., 1997) is related to the mean star of intersection of a RACS (Molchanov, 1997).

5. Variants of the contagion index can be constructed that express second-order and geometric properties of RACS.

A crucial contribution here is that LPIs can be viewed as estimators of underlying random processes. To the author's knowledge this is the first time non-parametric RACS summary functions have been explicitly correlated with LPI concepts. Kautz et al. (Kautz et al., 2011) provide an example of the power of non-parametric RACS very similar to LPI without directly referring to LPIs. Otherwise previous use of RACS in relation to LPIs have been for simulation studies (Hargis et al., 1998) and model fitting (Diggle, 1981; Sadler, 2006).

The remainder of this section discusses the core area, the closely related SCD, and a new contagion index that uses the SCD. The new SCD-based contagion is less resolution dependent than the classic pixel-adjacency contagion. For core area, the RACS perspective provides variance information, border corrections and natural conditioning on events. We will also make use of the coverage probability which is easily estimated by the percentage of landscape. The details for other indices will be discussed in a forthcoming paper.

In the following suppose that $X$ is a stationary, mixing RACS process, and that we have observed a single realisation, $X_{o b s}$, in a window $W$.

\subsection{Percentage of Core Area}

For a user chosen buffer distance, $r$, core area is the area within a class that is more than $r$ distance from the edge of the class (McGarigal, 2015; Didham and Ewers, 2012). The percentage of core area in a window is an estimate of the probability that a point will be further than $r$ distance from the exterior of $X$. This is also the probability of placing a disc of radius $r$ entirely within $X$,

$$
\text { Percentage of core area } \approx P\left(B_{r}(o) \subseteq X\right),
$$

where $B_{r}(o)$ is a disc of radius $r$ about the origin, $o$. The origin is used here arbitrarily because the stationarity of $X$ requires that the probability is the same regardless of the disc's centre. Thus the analogous concept of core area for a random process could be described as Core Probability and is the probability that a point will be further than $r$ distance from the exterior of $X$. In the next section (Section 3.1.1) we show that core probability is closely related to the SCD.
3.1.1 Core Probability and the SCD. The SCD (also known as the empty space function) is a popular tool for exploration and inference of random point processes (Baddeley et al., 2015) and sets (Diggle, 1981; Molchanov, 1997; Heinrich, 1993). The unconditional version is the probability of $X$ intersecting an arbitrarily located disc of radius $r$,

$$
S C D_{X}^{u n c}(r)=P\left(B_{r}(o) \cap X \neq \emptyset\right) .
$$

where $\emptyset$ is the empty set so ' $\neq \emptyset$ ' can be read as 'not empty' and we have arbitrarily used the origin as the centre of the disc.

The conditional version of the SCD is the probability of $X$ intersecting a disc given that the centre of the disc is not in $X$,

$$
S C D_{X}^{c o n d}(r)=P\left(B_{r}(o) \cap X \neq \emptyset \mid o \notin X\right) .
$$

If $o \in X$ then $X$ intersects the disc so the two versions are related by

$$
S C D_{X}^{\text {cond }}(r)=1-\frac{1-S C D_{X}^{u n c}(r)}{1-p},
$$

where $p$ is the coverage probability. Recall that the coverage probability is the probability of an arbitrary point being in $X$, it can be estimated by the percentage of $W$ covered by $X_{o b s}$ (Baddeley and Jensen, 2004).

The SCD describes the sizes of space outside $X$; a RACS with a large conditional SCD at radius $r$ is less likely to contain gaps in which a disc of radius $r$ can fit.

The space that is not interior to $X$ is also a RACS, we denote it by $\overline{X^{c}}$. The superscript ' $c$ ' denotes the set of locations not in $X$ and the overline represents the inclusion of the edges. If $X$ is stationary then so is $\overline{X^{c}}$ and thus the SCD of $\overline{X^{c}}$ is well defined. Furthermore the unconditional SCD of $\overline{X^{c}}$ is the probability that an arbitrarily located disc of radius $r$ intersects $\overline{X^{c}}$ which is the negation of the core probability. In other words

$$
\begin{aligned}
S C D_{\overline{X^{c}}}^{\text {unc }}(r) & =P\left(B_{r}(o) \cap \overline{X^{c}} \neq \emptyset\right) \\
& =P\left(B_{r}(o) \nsubseteq X\right) \\
& =1-\text { core probability. }
\end{aligned}
$$

The conditional version is the probability that a point in $X$ is within distance $r$ of the outside of $X$

$$
S C D_{\bar{X}^{c}}^{\text {cond }}(r)=P\left(B_{r}(o) \cap \overline{X^{c}} \neq \emptyset \mid o \notin \overline{X^{c}}\right)
$$

which suggests a conditional core probability (CCP), the probability that a point in $X$ is in the core of $X$,

$$
\begin{aligned}
\text { conditional core probability } & =1-S C D_{X^{c}}^{\text {cond }}(r) \\
& =P\left(B_{r}(0) \subseteq X \mid o \in X\right) .
\end{aligned}
$$

Thus the non-parametric properties of the unconditional and conditional SCD are identical to those of the core probability and the CCP respectively.

3.1.2 Border Correction. Estimation of the core probability using percentage of core area risks making an implicit assumption about $X$ outside the observation window. See Figure 2. There are points which appear further than $r$ distance from the edge of $X_{o b s}$ but may not be in the core of $X$. The same is true for the SCD. The effect is 
larger with larger buffer distance because the area within $r$ distance of the boundary is a larger proportion of the window area. We mention three different border corrected estimators for the unconditional SCD that are available in the spatstat package within the $\mathrm{R}$ statistical computing environment (Baddeley et al., 2015). For brevity we describe them only in terms of the core probability. Despite theoretical differences the three estimators perform similarly well in simulation experiments for a variety of random patterns of points (Stoyan, 2006; Baddeley et al., 2015), which suggests that they will also perform with similar quality on RACS. Note that the CCP can be estimated from the ratio of a core probability estimate and a coverage probability estimate.

The reduced sample estimate uses only those points further than $r$ distance from the window's boundary (Heinrich, 1993). See Figure 2. Due to the different sample size for each $r$ the estimated core probability can increase as the buffer distance increases, however an increase in the true core probability is not possible because a point not in the core of $X$ for buffer distance $r$ cannot be in the core for larger buffer distances.

The Chiu-Stoyan correction uses the length of the set of points exactly $k$ distance from the boundary of $X_{o b s}$ and integrates $k$ between 0 and the desired radius $r$. It produces estimates for each radius $r$ that are unbiased and can not increase with $r$, but there is a chance that the estimate will pass below 0 (Chiu and Stoyan, 1998).

Alternatively the Kaplan-Meier correction, which uses methods for the analysis of censored survival times (Baddeley and Gill, 1994), also provides non-increasing estimates of the core probability.

3.1.3 Variance. For each radius, $r$, the reduced sample estimator is similar to the coverage probability estimator. Molchanov (Molchanov, 1997, §4.3) provides a variance if certain properties of the process are known a priori. It may also be possible to estimate the variance using spectral density (Mase, 1982; Böhm et al., 2004), however this method requires a suitable choice of smoothing bandwidth.

\subsection{Disc-State Contagion}

Contagion is a popular entropy-inspired LPI for describing aggregation of classes. The unnormalised version is defined as (O’Neill et al., 1988; Li and Reynolds, 1993)

$$
\text { Contagion }:=\sum_{i=1}^{m} \sum_{j=1}^{m} P_{i j} \ln \left(P_{i j}\right),
$$

where $m$ is the number of classes and $P_{i j}$ is the probability of randomly selected adjacent pixels being in class $i$ and class $j$ respectively. Although it was initially designed for multiple categories we restrict our focus to two-class maps.

Contagion as it is defined above describes the aggregation of classes within a distance of double the ground sample distance (double the ground sample distance because pixels are typically an average or weighted integral of the corresponding sample region). Contagions calculated at different resolutions thus describe aggregation at different scales making contagion very sensitive to resolution changes. Moreover because the definition depends on the resolution of an observation technique there is no canonical

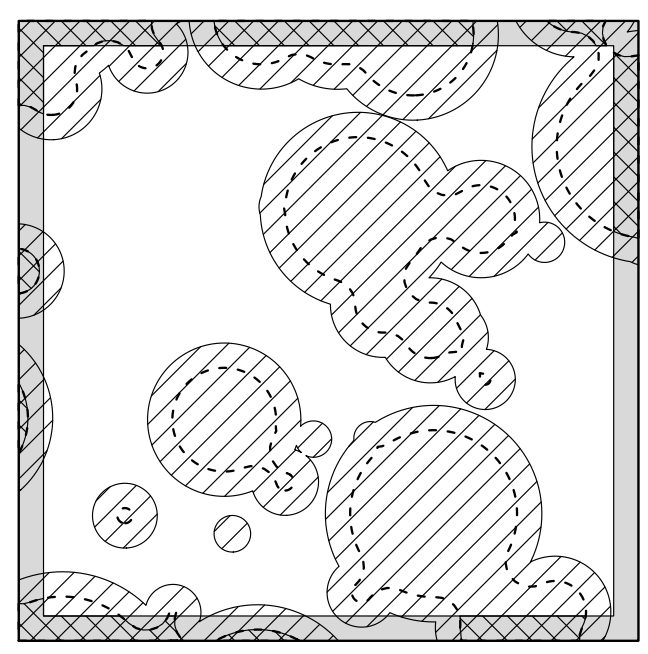

Figure 2: An example observation of a RACS, $X_{\text {obs }}$ (hatched regions), showing points within $r$ distance of the window boundary (grey region) and points that are further than $r$ distance from the outside of $X_{o b s}$ (dashed boundary). The crosshatched regions are within $r$ distance of the edge of $W$ and further than $r$ distance from the visible edge of $X_{\text {obs }}$. Without more information it is impossible to know whether these points are in the core area of $X$. Reduced sample Estimator: The reduced sample estimator for the core probability is the proportion of the non-grey region that is inside the dashed boundary. Chiu-Stoyan Estimator: The Chiu-Stoyan estimator uses the length of the dashed boundary outside the grey region given by multiple smaller buffer distances to estimate the core probability at the desired buffer distance.

definition for the contagion of a real landscape. A number of other metrics that use the same pixel adjacency concept have similar issues.

Ramezani and Holm (Ramezani and Holm, 2011) encountered this issue when they tried to apply contagion to polygonal data. Instead they considered the adjacency probabilities $P_{i j}$ to be the probability of class $j$ intersecting a circle of radius $r$ around a point in class $i$. Thus contagion became a functional metric (a function of the radius $r$ ).

We present another variant of contagion that describes the mixing of classes within a disc, termed disc-state contagion. Let 1 denote inside $X$ and 0 denote outside $X$. We define $P_{10}(r)$ as the probability that a point is in $X$ and not in the core of $X$,

$$
P_{10}(r)=P\left(o \in X, B_{r}(o) \nsubseteq X\right),
$$

and $P_{01}(r)$ as the probability that a point is in $\overline{X^{c}}$ and not in the core of $\overline{X^{c}}$,

$$
P_{01}(r)=P\left(o \in \overline{X^{c}}, B_{r}(o) \nsubseteq \overline{X^{c}}\right) .
$$

We define the remaining elements, $P_{11}(r)$ as the core probability of $X$,

$$
P_{11}(r):=P\left(B_{r}(o) \subseteq X\right),
$$

and similarly $P_{00}$ as the core probability of $\overline{X^{c}}$.

For each radius $r$, this version of contagion quantifies the 
disorder of a system with four states (1) the entire disc is in $X,(2)$ the entire disc is outside $X,(3)$ the centre is within $X$ and some of the disc is outside $X$, and (4) the centre is outside $X$ and some of the disc is inside $X$. A diagram of each state is in Figure 3. The lowest contagion (and highest entropy) occurs when the probability of each of these states is $\frac{1}{4}$.

This disc-state contagion can be estimated using estimates of the SCD and core probability. It is well defined for any stationary RACS, and because the disc size is not linked to resolution it is more robust to resolution changes than the classic pixel-adjacency contagion (9).

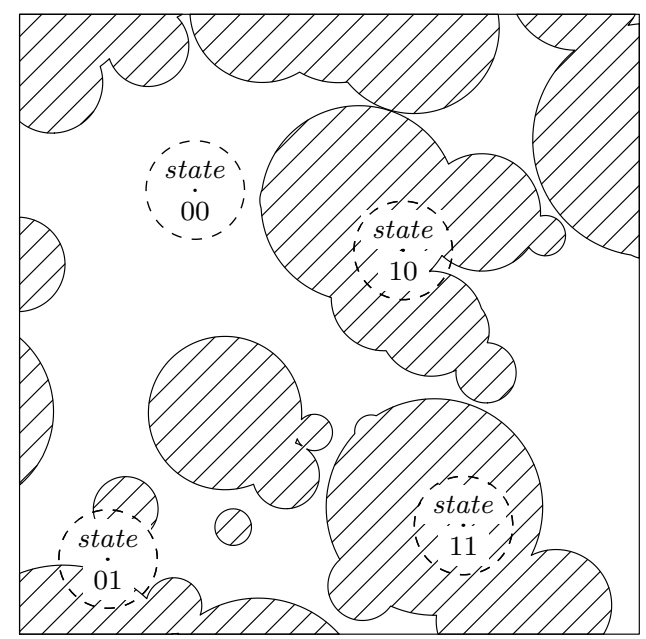

Figure 3: Examples of each disc state in disc-state contagion. Hatched regions denote locations inside the realisation of $X$.

\section{EXPLORATION OF TREE CANOPY PATTERN PROCESSES AND FEED QUALITY}

We explored decimetre resolution maps of tree canopy in Perth, Australia, using conditional SCD, CCP, disc-state contagion and coverage probability. The canopy height maps were derived from stereo photography through height estimates (stereo matching) and spectral values (Caccetta et al., 2015). Two-class categorical maps were then obtained by only keeping canopy that was higher than $4 m$.

We compared the RACS estimators with field-based analysis of feed quality of Banskia woodlands for an endangered bird (van Dongen et al., 2016). For each location of the field-based assessment we used a circular window of $30 m$ radius to estimate RACS properties. We randomly selected $50 \%$ of these field-assessed locations for initial exploration, reserving the remaining $50 \%$ for validation.

In making these estimates it was assumed that each window observed a stationary and mixing tree canopy process. Given little information on covariates such as soil, moisture and wind this is a tolerable representation for a first analysis.

The best gradation of feed quality was obtained by the CCP. A small cluster of very high feed quality processes appeared at core buffer distances of $0.75 \mathrm{~m}$ and above (Figure 4). In comparison the coverage probability could not separate very high feed locations from moderate feed locations (Figure 5). The SCD and disc-state contagion did not show any obvious association to feed quality (Figure $6)$.

Unfortunately the above discrimination of high feed locations did not generalise to the validation data (Figure 7). Further exploration of other RACS summary functions and different canopy heights might uncover real associations. Discrimination might also be achieved through multi-type RACS (with each class corresponding to a different height range), 3D stochastic models, or non-stationary RACS.

Conditional Core Probability and Feed Quality

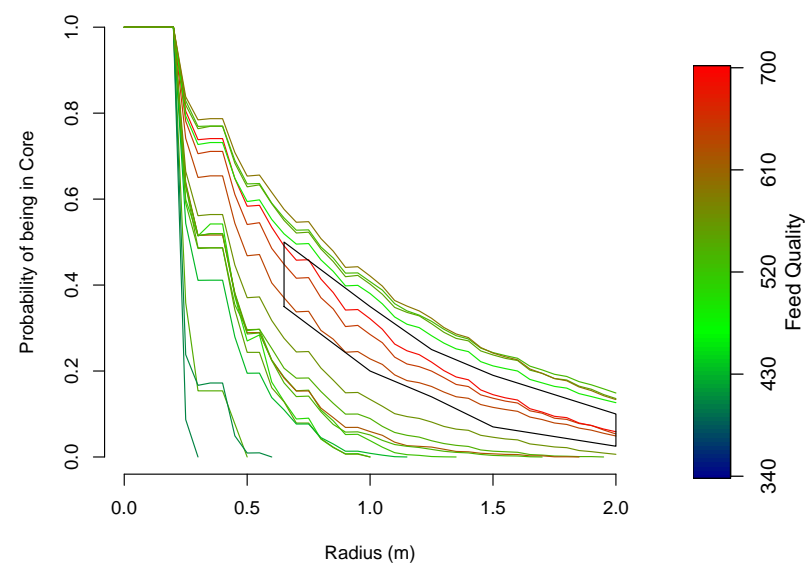

Figure 4: Conditional core probability for tree canopy above $4 \mathrm{~m}$. Each location/observation is a different curve. The colours correspond to feed quality (see colour bar on right). The canopy processes with the three highest feed quality scores (in red) are clustered together for buffer distances from $0.75 m$ to $2 m$ (black polygon). Reduced sample boundary correction was used here but the effect was minor at these distances (see Section 4.1 and Figure 8). Note that the steps in the functions were due to the map resolution of $0.2 \mathrm{~m}$, and the slight upward directions of some of these jumps was caused by the reduced sample correction.

\subsection{Effect of Border Correction}

Border correction had little impact on the above relationship because the buffer distances involved were small $(2 \mathrm{~m})$ compared to the window diameter $(60 \mathrm{~m})$ (Figure 8). However the impact of border correction on the SCD (Figure 9) suggests that CCP estimates would have been significantly impacted if the canopy patches were much larger (e.g. tens of metres in diameter).

\section{COMPUTATION}

Computations were performed inside the $\mathrm{R}$ statistical environment (R Core Team, 2016) on a $3.10 \mathrm{GHz}$ CPU with $4 \mathrm{~GB}$ of RAM. The spatstat package (Baddeley et al., 2015) was used heavily with tools for reading remotely sensed data provided by the raster package (Hijmans, 2015) and GDAL (GDAL Development Team, 2015). An R package containing additional necessary functions is available from the author.

For the $30 \mathrm{~m}$ radius observations above, estimation of the SCD, CCP, disc-state and coverage probability usually required 0.6 seconds of computation. For larger windows of 


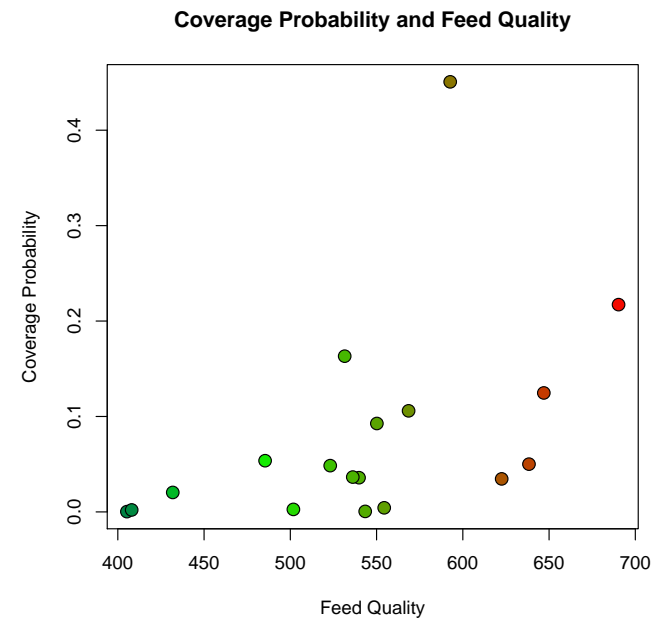

Figure 5: Coverage probability of canopy above $4 m$ and feed quality. Each point is a different location. Note that high coverage probability suggests higher feed value, but coverage probability doesn't discriminate between high feed locations and moderate feed locations.

1500 by 1500 pixels $(300 m$ by $300 m$ ) performing the same estimations required less than 10 seconds.

\section{CONCLUSION}

RACS provide a powerful generic framework for modelling the processes underlying categorical maps. They are especially useful at high resolutions where the geometries created by patches of many pixels are important. The RACS framework provides a conceptual tool to guide LPI use and design including the treatment of sensing artefacts such as resolution and map extent. Here we focused on non-parametric RACS tools under the assumptions of stationarity and mixing. These tools are stochastic, processcentric versions, of LPI and we discussed the core area LPI in detail.

Core area is closely related to the spherical contact distribution. We first linked core area to a probabilistic concept, the core probability, and then showed that core probability was the opposite of the spherical contact distribution evaluated at a particular radius. This lead to functional versions of the core probability, a conditional core probability, and border corrected estimators.

The well-defined spherical contact distribution also suggested a resolution-free version of the contagion LPI. This new version of contagion describes the entropy of the state of a disc and its centre. The aggregation scale that this new contagion expresses is chosen by the user and is independent of the imaging resolution. In comparison the original pixel-adjacency contagion describes interaction over the width of two pixels and is thus much more sensitive to resolution change.

A preliminary exploration of tree canopy processes briefly demonstrated core probability, the spherical contact distribution, disc-state contagion and border corrections. Due to time restrictions the demonstrations did not include hypothesis tests.
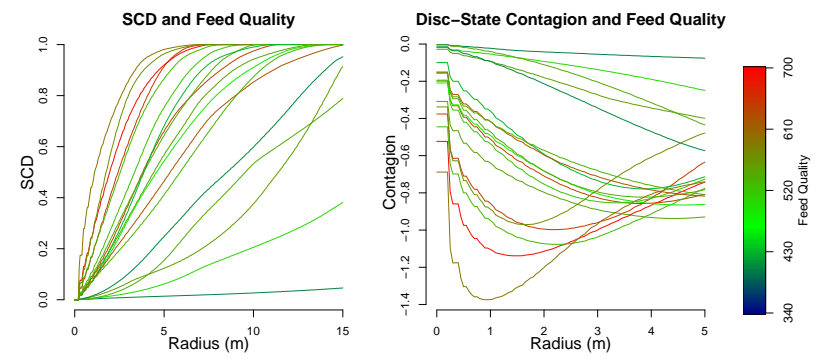

Figure 6: Conditional SCD (left) and disc-state contagion (right). Curves are coloured by feed quality. There is no discernible pattern of high feed quality processes.

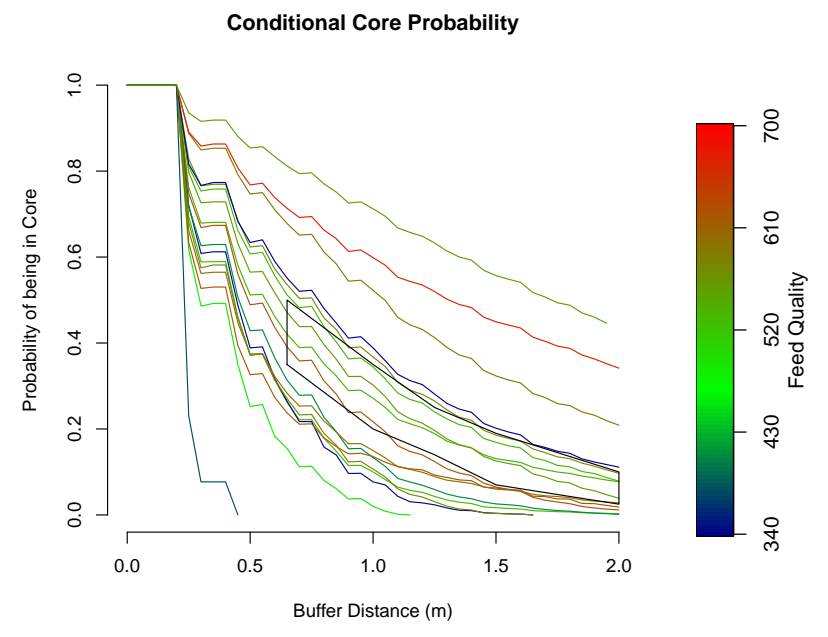

Figure 7: CCP for the validation data. The region of high feed process observed in the exploration data is shown (black polygon). The separation did not generalise to the validation data.

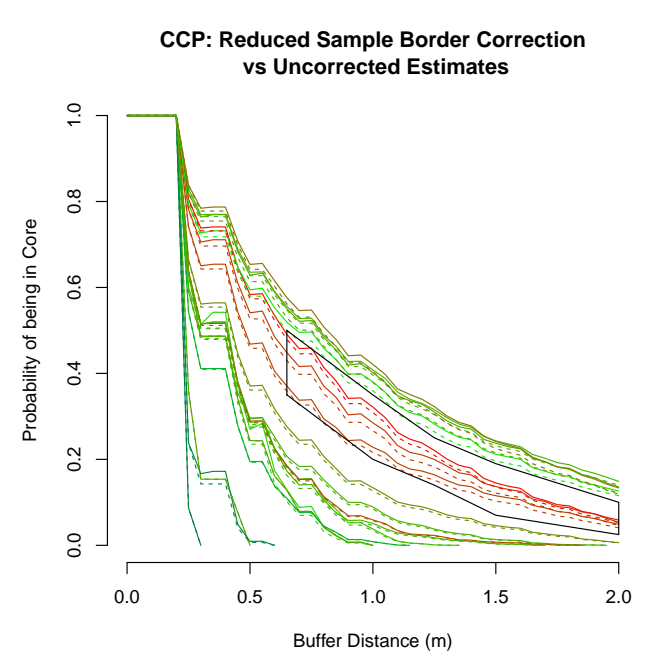

Figure 8: The reduced sample CCP estimates (solid lines) and uncorrected CCP estimates (dashed lines). At these small buffer distances (relative to window size) border correction has minor impact. Note that the uncorrected estimates are lower because they have implicitly assumed that no tree canopy existed outside the observation window. 
SCD: Impact of Reduced Sample Correction

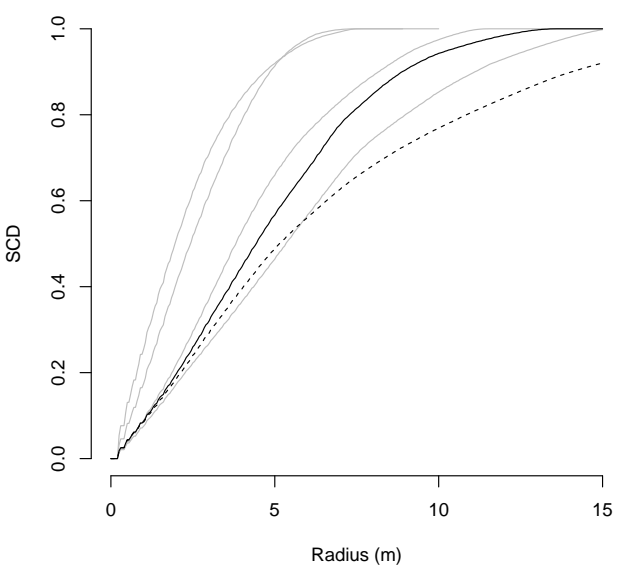

Figure 9: The SCD estimate for one location using reduced sample correction (solid line) and no border correction (dashed line). For context the reduced sample estimates of some other observations are shown in grey. Note that, similar to the uncorrected estimates of CCP, the uncorrected estimate of SCD is lower because it has implicitly assumed that no tree canopy existed outside the observation window. For some observations, such as the one shown here, the difference between border corrected and uncorrected estimates can be quite large. Thus using the uncorrected estimates could have serious implications for applications of the SCD.

\section{ACKNOWLEDGEMENTS}

Many thanks to Australia's Commonwealth Scientific and Industrial Research Organisation for supplying the tree canopy maps, and to Ricky van Dongen and Geoff Barrett of Western Australia's Dept. of Parks and Wildlife for providing the feed data.

I would also like to thank my $\mathrm{PhD}$ supervisors Adrian Baddeley, Peter Caccetta, and Gopalan Nair.

\section{References}

Ayala, G. and Simó, A., 1995. Bivariate random closed sets and nerve fibre degeneration. Advances in Applied Probability 27(2), pp. 293-305.

Baddeley, A. and Gill, R. D., 1994. The empty space hazard of a spatial pattern. Technical report, Department of Mathematics, University of Utrecht. Preprint 845.

Baddeley, A. and Jensen, E. B. V., 2004. Stereology for Statisticians. Monographs on Statistics \& Applied Probability, Vol. 103, Chapman and Hall/CRC, Boca Raton, Florida.

Baddeley, A., Rubak, E. and Turner, R., 2015. Spatial Point Patterns: Methodology and Applications with $R$. Chapman and Hall/CRC, London.

Barbour, A. D. and Schmidt, V., 2001. On Laslett's transform for the Boolean model. Advances in Applied Probability 33(1), pp. 1-5.
Böhm, S., Heinrich, L. and Schmidt, V., 2004. Kernel estimation of the spectral density of stationary random closed sets. Australian \& New Zealand Journal of Statistics 46(1), pp. 41-51.

Caccetta, P., Collings, S., Devereux, A., Hingee, K., McFarlane, D., Traylen, A., Wu, X. and Zhou, Z., 2015. Monitoring land surface and cover in urban and periurban environments using digital aerial photography. International Journal of Digital Earth pp. 1-19.

Chang, Y., Baddeley, A., Wallace, J. and Canci, M., 2013. Spatial statistical analysis of tree deaths using airborne digital imagery. International Journal of Applied Earth Observation and Geoinformation 21, pp. 418-426.

Chiu, S. N. and Stoyan, D., 1998. Estimators of distance distributions for spatial patterns. Statistica Neerlandica 52(2), pp. 239-246.

Chiu, S. N., Stoyan, D., Kendall, W. S. and Mecke, J., 2013. Stochastic Geometry and Its Applications. 3 edn, John Wiley \& Sons.

Cressie, N., Assuncao, R., Holan, S., Levine, M., Nicolis, O., Zhang, J. and Zou, J., 2012. Dynamical random-set modeling of concentrated precipitation in North America. Statistics and its Interface 5(2), pp. 169-182.

Cushman, S. A., McGarigal, K. and Neel, M. C., 2008. Parsimony in landscape metrics: Strength, universality, and consistency. Ecological Indicators 8(5), pp. 691-703.

Daley, D. and Vere-Jones, D., 2008. An Introduction to the Theory of Point Processes II: General Theory and Structure. Probability and its applications, Vol. 2, 2 edn, Springer Science, New York, N.Y., United States.

Descombes, X. (ed.), 2012. Stochastic Geometry for Image Analysis. ISTE / John Wiley \& Sons.

Didham, R. K. and Ewers, R. M., 2012. Predicting the impacts of edge effects in fragmented habitats: Laurance and Yensen's core area model revisited. Biological Conservation 155, pp. 104-110.

Diggle, P. J., 1981. Binary mosaics and the spatial pattern of heather. Biometrics 37(3), pp. 531-539.

Dramstad, W. E., 2009. Spatial metrics - useful indicators for society or mainly fun tools for landscape ecologists? Norsk Geografisk Tidsskrift - Norwegian Journal of Geography 63(4), pp. 246-254.

Fortin, M. and Agrawal, A. A., 2005. Landscape ecology comes of age. Ecology 86(8), pp. 1965-1966.

Fortin, M. J., Boots, B., Csillag, F. and Remmel, T. K., 2003. On the role of spatial stochastic models in understanding landscape indices in ecology. Oikos 102(1), pp. 203-212.

GDAL Development Team, 2015. GDAL - geospatial data abstraction library. Version 2.0.0 http://www.gdal.org (3 Mar. 2016).

Hargis, C. D., Bissonette, J. A. and David, J. L., 1998. The behavior of landscape metrics commonly used in the study of habitat fragmentation. Landscape Ecology 13(3), pp. 167-186. 
Heinrich, L., 1993. Asymptotic properties of minimum contrast estimators for parameters of Boolean models. Metrika 40(1), pp. 67-94.

Hijmans, R. J., 2015. raster: Geographic data analysis and modeling. Version 2.5-2 http://CRAN.Rproject.org/package $=$ raster (3 Apr. 2016).

Hug, D., Last, G. and Weil, W., 2002. A survey on contact distributions. In: K. Mecke and D. Stoyan (eds), Morphology of Condensed Matter, Lecture Notes in Physics, Springer Berlin Heidelberg, pp. 317-357.

Kautz, M., Düll, J. and Ohser, J., 2011. Spatial dependence of random sets and its application to disperion of bark beetle infestation in a natural forest. Image Analysis 85 Stereology 30(3), pp. 123-131.

Keitt, T. H., Urban, D. L. and Milne, B. T., 1997. Detecting critical scales in fragmented landscapes. Conservation ecology 1(1), pp. 4.

Kupfer, J. A., 2012. Landscape ecology and biogeography: Rethinking landscape metrics in a post-FRAGSTATS landscape. Progress in Physical Geography 36(3), pp. $400-420$.

Li, H. and Reynolds, J. F., 1993. A new contagion index to quantify spatial patterns of landscapes. Landscape Ecology 8(3), pp. 155-162.

Lustig, A., Stouffer, D. B., Roigé, M. and Worner, S. P., 2015. Towards more predictable and consistent landscape metrics across spatial scales. Ecological Indicators 57, pp. 11-21.

Mase, S., 1982. Asymptotic properties of stereological estimators of volume fraction for stationary random sets. Journal of Applied Probability 19(1), pp. 111-126.

Matheron, G., 1975. Random sets and integral geometry. John Wiley \& Sons, USA.

McGarigal, K., 2015. FRAGSTATS help. Technical report, University of Massachusetts. http://www.umass.edu/landeco/research/fragstats/ documents/fragstats_documents.html (12 May 2015).

Molchanov, I., 1984. Labelled random sets. Theory of Probability and Mathematical Statistics 29, pp. 113-119.

Molchanov, I., 1997. Statistics of the Boolean Model for Practitioners and Mathematicians. John Wiley \& Sons.

Molchanov, I. S., 2005. Theory of random sets. Probability and its applications, Springer, London.

O’Neill, R. V., Krummel, J. R., Gardner, R. H., Sugihara, G., Jackson, B., DeAngelis, D. L., Milne, B. T., Turner, M. G., Zygmunt, B., Christensen, S. W., Dale, V. H. and Graham, R. L., 1988. Indices of landscape pattern. Landscape Ecology 1(3), pp. 153-162.

R Core Team, 2016. R: A language and environment for statistical computing. Vienna, Austria www.Rproject.org (3 Apr. 2016).

Ramezani, H. and Holm, S., 2011. A distance dependent contagion function for vector-based data. Environmental and Ecological Statistics 19(2), pp. 161-181.
Remmel, T. K. and Csillag, F., 2003. When are two landscape pattern indices significantly different? Journal of Geographical Systems 5(4), pp. 331-351.

Rossi, J., Garcia, J., Roques, A. and Rousselet, J., 2015. Trees outside forests in agricultural landscapes: Spatial distribution and impact on habitat connectivity for forest organisms. Landscape Ecology 31(2), pp. 243-254.

Sadler, R., 2006. Image-based modelling of pattern dynamics in a semiarid grassland of the Pilbara, Australia. $\mathrm{PhD}$, School Of Plant Biology, University of Western Australia, Perth, Australia. (Thesis).

Schindler, S., Poirazidis, K. and Wrbka, T., 2008. Towards a core set of landscape metrics for biodiversity assessments: A case study from Dadia National Park, Greece. Ecological Indicators 8(5), pp. 502-514.

Schröder, B. and Seppelt, R., 2006. Analysis of pattern-process interactions based on landscape models - Overview, general concepts, and methodological issues. Ecological Modelling 199(4), pp. 505-516.

Stoyan, D., 2006. On estimators of the nearest neighbour distance distribution function for stationary point processes. Metrika 64(2), pp. 139-150.

Stoyan, D. and Mecke, K., 2005. The boolean model: from matheron till today. In: M. Bilodeau, F. Meyer and M. Schmitt (eds), Space, Structure and Randomness, Lecture Notes in Statistics, Springer New York, pp. 151-181.

Turner, M. G., 2005. Landscape ecology: What is the state of the science? Annual Review of Ecology, Evolution, and Systematics 36, pp. 319-344.

van Dongen, R., Barrett, G., Zdunic, K., Huntley, B. and Mitchell, D., 2016. Food potential and night roost mapping for calyptorhynchus latirostris using digital aerial photography. Technical report, Dept. of Parks and Wildlife, Perth, Australia. in prep.

VanDerWal, J., Falconi, L., Januchowski, S., Shoo, L. and Storlie, C., 2015. Package 'SDMTools': Species distribution modelling tools: Tools for processing data associated with species distribution modelling exercises. Version 1.1-221 http://cran.rproject.org/package=SDMTools (2 Apr 2016).

Wang, X. and Cumming, S. G., 2011. Measuring landscape configuration with normalized metrics. Landscape Ecology 26(5), pp. 723-736.

Zhao, X., Stein, A. and Chen, X., 2009. Application of random sets to model uncertainties of natural entities extracted from remote sensing images. Stochastic Environmental Research and Risk Assessment 24(5), pp. 713723. 